\title{
2019 update to: Management of hyperglycaemia in type 2 diabetes, 2018. A consensus report by the American Diabetes Association (ADA) and the European Association for the Study of Diabetes (EASD)
}

\author{
John B. Buse ${ }^{1}$ (1) - Deborah J. Wexler ${ }^{2,3}$ (D) $\cdot$ Apostolos Tsapas $^{4}$ (D) Peter Rossing ${ }^{5,6} \cdot$ Geltrude Mingrone $^{7,8,9}$ (D) \\ Chantal Mathieu ${ }^{10}$ (D) David A. D'Alessio ${ }^{11} \cdot$ Melanie J. Davies $^{12}$ (1)
}

Published online: 19 December 2019

(C) European Association for the Study of Diabetes and American Diabetes Association 2019

\begin{abstract}
The American Diabetes Association and the European Association for the Study of Diabetes have briefly updated their 2018 recommendations on management of hyperglycaemia, based on important research findings from large cardiovascular outcomes trials published in 2019. Important changes include: (1) the decision to treat high-risk individuals with a glucagon-like-peptide 1 (GLP-1) receptor agonist or sodium-glucose cotransporter 2 (SGLT2) inhibitor to reduce major adverse cardiovascular events (MACE), hospitalisation for heart failure (hHF), cardiovascular death or chronic kidney disease (CKD) progression should be considered independently of baseline $\mathrm{HbA}_{1 \mathrm{c}}$ or individualised $\mathrm{HbA}_{1 \mathrm{c}}$ target; (2) GLP-1 receptor agonists can also be considered in patients with type 2 diabetes without established cardiovascular disease (CVD) but with the presence of specific indicators of high risk; and (3) SGLT2 inhibitors are recommended in patients with type 2 diabetes and heart failure, particularly those with heart failure with reduced ejection fraction, to reduce hHF, MACE and CVD death, as well as in patients with type 2 diabetes with CKD (eGFR 30 to $\leq 60 \mathrm{ml} \mathrm{min}^{-1}[1.73 \mathrm{~m}]^{-2}$ or urinary albumin-to-creatinine ratio $>30 \mathrm{mg} / \mathrm{g}$, particularly $>300 \mathrm{mg} / \mathrm{g}$ ) to prevent the progression of CKD, hHF, MACE and cardiovascular death.
\end{abstract}

Keywords Cardiovascular disease · Chronic kidney disease · Glucose-lowering therapy · Guidelines · Heart failure · Hypoglycaemia $\cdot$ Patient-centred care $\cdot$ Type 2 diabetes mellitus

M. J. Davies and J. B. Buse were co-chairs for the Consensus Statement Writing Group. D. D'Alessio and D. J. Wexler were the writing group members for the ADA. C. Mathieu, G. Mingrone, P. Rossing and A. Tsapas were the writing group members for the EASD.

This article is being simultaneously published in Diabetologia (https:// doi.org/10.1007/s00125-019-05039-w) and Diabetes Care (https://doi. org/10.2337/dci19-006) by the European Association for the Study of Diabetes and the American Diabetes Association.

Melanie J. Davies

melanie.davies@uhl-tr.nhs.uk

1 Department of Medicine, University of North Carolina School of Medicine, Chapel Hill, NC, USA

2 Department of Medicine and Diabetes Unit, Massachusetts General Hospital, Boston, MA, USA

3 Harvard Medical School, Boston, MA, USA

4 Second Medical Department, Aristotle University Thessaloniki, Thessaloniki, Greece

5 Steno Diabetes Center Copenhagen, Gentofte, Denmark

6 University of Copenhagen, Copenhagen, Denmark
Fondazione Policlinico Universitario A. Gerelli IRCCS, Roma, Italia

8 Università Cattolica del Sacro Cuore, Roma, Italia

9 Diabetes and Nutritional Sciences, King's College London, London, UK

10 Clinical and Experimental Endocrinology, UZ Gasthuisberg, KU Leuven, Leuven, Belgium

11 Department of Medicine, Duke University School of Medicine, Durham, NC, USA

12 Diabetes Research Centre, University of Leicester, Leicester General Hospital, Leicester, UK 


\begin{tabular}{|c|c|}
\hline \\
\hline \multirow{2}{*}{ CANVAS } & Canagliflozin Cardiovascular \\
\hline & Assessment Study \\
\hline CKD & Chronic kidney disease \\
\hline \multirow[t]{3}{*}{ CREDENCE } & Canagliflozin and Renal Events in \\
\hline & Diabetes with Established \\
\hline & Nephropathy Clinical Evaluation \\
\hline $\mathrm{CV}$ & Cardiovascular \\
\hline CVD & Cardiovascular disease \\
\hline CVOT & Cardiovascular outcomes trial \\
\hline \multirow[t]{2}{*}{ DAPA-HF } & Dapagliflozin and Prevention of \\
\hline & Adverse Outcomes in Heart Failure \\
\hline DPP-4 & Dipeptidyl peptidase-4 \\
\hline \multirow{2}{*}{ DECLARE-TIMI 58} & Dapagliflozin Effect on \\
\hline & $\begin{array}{l}\text { Cardiovascular Events-Thrombolysis } \\
\text { in Myocardial Infarction } 58\end{array}$ \\
\hline $\mathrm{EF}$ & Ejection fraction \\
\hline GLP-1 & Glucagon-like peptide 1 \\
\hline GLP-1 RA & GLP-1 receptor agonist \\
\hline $\mathrm{HF}$ & Heart failure \\
\hline HFrEF & $\begin{array}{l}\text { Heart failure with reduced ejection } \\
\text { fraction }\end{array}$ \\
\hline hHF & Hospitalisation for heart failure \\
\hline MACE & Major adverse cardiovascular event \\
\hline REWIND & $\begin{array}{l}\text { Researching Cardiovascular Events } \\
\text { with a Weekly Incretin in Diabetes }\end{array}$ \\
\hline SGLT2 & Sodium-glucose cotransporter 2 \\
\hline SGLT2i & SGLT2 inhibitor \\
\hline UACR & Urinary albumin-to-creatinine ratio \\
\hline
\end{tabular}

The American Diabetes Association (ADA) and the European Association for the Study of Diabetes (EASD) requested a brief update of the 2018 recommendations on management of hyperglycaemia [1,2], based on the important research findings published in 2019, with a particular focus on new data from large cardiovascular outcomes trials (CVOTs). The authors began work on the brief update in July 2019 and submitted it for publication in Diabetes Care and Diabetologia in October 2019. Work was conducted over a series of phone calls and by electronic interactions. This brief update provides a summary of the implications of this new evidence on recommendations for the management of hyperglycaemia in type 2 diabetes (see text box), which will be addressed more fully in the ADA Standards of Medical Care in Diabetes - 2020 (https://professional.diabetes.org/ SOC). It should be considered in conjunction with the 2018 consensus report $[1,2]$.

The Researching Cardiovascular Events with a Weekly Incretin in Diabetes (REWIND) trial of the glucagon-like peptide 1 (GLP-1) receptor agonist dulaglutide included a greater proportion of individuals with type 2 diabetes with high cardiovascular risk but without prior established cardiovascular disease (CVD) $(68.5 \%)$ and with longer follow-up (median 5.4 years) than prior CVOTs [3]. The primary major adverse cardiovascular event (MACE) outcome occurred in 2.7 per 100 patient-years with an HR of 0.88 (95\% CI $0.79,0.99)$ in favour of dulaglutide. There was no difference in the MACE effect in the subpopulations with and without a history of CVD, although the treatment effect of dulaglutide did not reach statistical significance when the groups were considered separately. Most other CVOTs with GLP-1 receptor agonists have included a minority of patients with risk factors only but without evidence of benefit on MACE outcomes in the lower-risk subgroups. Whether the differences in outcomes in trial subgroups without established CVD are related to study details or to the assigned therapy is uncertain. In REWIND, prior CVD was defined as a history of myocardial infarction, ischaemic stroke, unstable angina with ECG changes, myocardial ischaemia on imaging or stress test, or coronary, carotid or peripheral revascularisation. We previously recommended that established CVD was a compelling indication for treatment with a GLP-1 receptor agonist or sodium-glucose cotransporter 2 (SGLT2) inhibitor. We now also suggest that to reduce risk of MACE, GLP-1 receptor agonists can also be considered in patients with type 2 diabetes without established CVD with indicators of high risk, specifically, patients aged 55 years or older with coronary, carotid or lower extremity artery stenosis $>\mathbf{5 0 \%}$, left ventricular hypertrophy, an eGFR $<60 \mathrm{ml} \mathrm{min}^{-1}$ $[1.73 \mathrm{~m}]^{-2}$ or albuminuria. To date, the level of evidence to support the use of GLP-1 receptor agonists for primary prevention is strongest for dulaglutide but lacking for other GLP-1 receptor agonists.

The Dapagliflozin Effect on Cardiovascular EventsThrombolysis in Myocardial Infarction 58 (DECLARETIMI 58) trial compared the SGLT2 inhibitor dapagliflozin with placebo and also enrolled a greater proportion of participants with type 2 diabetes without prior established CVD but with multiple risk factors (59.4\%) and with longer follow-up (median 4.2 years) than other SGLT2 inhibitor trials [4]. Dapagliflozin demonstrated cardiovascular (CV) safety but not a benefit for the MACE endpoint (HR 0.93, 95\% CI $0.84,1.03$ ). Dapagliflozin was associated with benefit for the co-primary efficacy endpoint of cardiovascular death or hospitalisation for heart failure (hHF) with HR $0.83(95 \% \mathrm{CI}$ $0.73,0.95$ ) as well as renal endpoints. For MACE, the HR in the multiple risk factor group without established atherosclerotic vascular disease was 1.01 , but this group had strong evidence for benefit for the composite of cardiovascular death or hHF. Meta-analysis of the SGLT2 inhibitor CVOTs suggest a class effect to reduce hHF and chronic kidney disease (CKD) progression across high and lower CVD risk subgroups with no effect on MACE in the absence of established atherosclerotic vascular disease [5]. 


\section{Changes to consensus recommendations}

We previously recommended that, in the setting of type 2 diabetes, established CVD was a compelling indication for treatment with a GLP-1 receptor agonist or SGLT2 inhibitor. We now further suggest the following:

\section{General consideration}

- In appropriate high-risk individuals with established type 2 diabetes, the decision to treat with a GLP-1 receptor agonist or SGLT2 inhibitor to reduce MACE, hHF, CV death or CKD progression should be considered independently of baseline $\mathrm{HbA}_{1 \mathrm{c}}$ or individualised $\mathrm{HbA}_{1 \mathrm{c}}$ target.

- Providers should engage in shared decision making around initial combination therapy in new-onset cases of type 2 diabetes.

\section{GLP-1 receptor agonist recommendations}

- For patients with type 2 diabetes and established atherosclerotic CV disease (such as those with prior myocardial infarction, ischaemic stroke, unstable angina with ECG changes, myocardial ischaemia on imaging or stress test, or revascularisation of coronary, carotid or peripheral arteries) where MACE is the gravest threat, the level of evidence for MACE benefit is greatest for GLP-1 receptor agonists.

- To reduce risk of MACE, GLP-1 receptor agonists can also be considered in patients with type 2 diabetes without established CVD with indicators of high risk, specifically, patients aged 55 years or older with coronary, carotid or lower extremity artery stenosis $>50 \%$, left ventricular hypertrophy, eGFR $<60$ $\mathrm{ml} \min ^{-1}\left[1.73 \mathrm{~m}^{-2}\right.$ or albuminuria.

\section{SGLT2 inhibitor recommendations}

- For patients with or without established atherosclerotic CVD, but with HFrEF (EF <45\%) or CKD (eGFR 30 to $\leq 60 \mathrm{ml} \mathrm{min}^{-1}\left[1.73 \mathrm{~m}^{-2}\right.$ or UACR $>30 \mathrm{mg} / \mathrm{g}$, particularly UACR $>300 \mathrm{mg} / \mathrm{g}$ ), the level of evidence for benefit is greatest for SGLT2 inhibitors.

- SGLT2 inhibitors are recommended in patients with type 2 diabetes and HF, particularly those with HFrEF, to reduce hHF, MACE and CV death.

- SGLT2 inhibitors are recommended to prevent the progression of CKD, hHF, MACE and CV death in patients with type 2 diabetes with CKD.

- Patients with foot ulcers or at high risk for amputation should only be treated with SGLT2 inhibitors after careful shared decision making around risks and benefits with comprehensive education on foot care and amputation prevention.

Analysis of two SGLT2 inhibitor CVOTs, DECLARETIMI 58 [6] and the Canagliflozin Cardiovascular Assessment Study (CANVAS) Program [7], suggests that the benefits of SGLT2 inhibitors for hHF, MACE and cardiovascular death are greatest for those individuals with preexisting heart failure with reduced ejection fraction (HFrEF) compared with those without HFrEF. It is important to note that hHF was a secondary outcome, relatively low numbers of patients had HF at baseline, and data on ejection fraction (EF) were only available for a proportion of patients. In DECLARE-TIMI 58, individuals with HF but no reduction of EF as well as those without HF did not seem to benefit from dapagliflozin treatment to lower MACE and cardiovascular death outcomes. The benefit for hHF was strongest for those who at baseline had an $\mathrm{EF}<30 \%$, strong for those with an $\mathrm{EF}$ $<45 \%$, and marginal for those with an $\mathrm{EF} \geq 45 \%$ or those without HF. The Dapagliflozin and Prevention of Adverse Outcomes in Heart Failure (DAPA-HF) trial of dapagliflozin was the first heart failure outcome trial of a diabetes medication [8]. Recruitment included patients with and without type 2 diabetes with heart failure and an $\mathrm{EF} \leq 40 \%$ and demonstrated benefits for reduction of the primary composite endpoint of $\mathrm{CV}$ death, hHF and urgent $\mathrm{HF}$ visits, as well as for HF events and mortality (CV and total) considered separately. We now suggest that SGLT2 inhibitors are recommended in patients with type 2 diabetes and HF, particularly those with HFrEF, to reduce hHF, MACE and CV death.

The REWIND trial of the GLP-1 receptor agonist dulaglutide had no lower limit to $\mathrm{HbA}_{1 \mathrm{c}}$ for eligibility and demonstrated equivalent efficacy for reduction of MACE above and below the median $\mathrm{HbA}_{1 \mathrm{c}}$ of $55 \mathrm{mmol} / \mathrm{mol}(7.2 \%)$ [3]. None of the CVOTs of SGLT2 inhibitors with primary MACE endpoints have recruited patients with an $\mathrm{HbA}_{1 \mathrm{c}}$ $<48 \mathrm{mmol} / \mathrm{mol}(<6.5 \%)$, and there is little data to inform clinical decision making for patients with an $\mathrm{HbA}_{1 \mathrm{c}}<53 \mathrm{mmol} /$ mol $(<7 \%)$ [9]. However, the outcome benefits observed in the CVOTs do not appear restricted to patients with an elevated $\mathrm{HbA}_{1 \mathrm{c}}$. That said, the DAPA-HF trial recruited patients with 
HFrEF with and without diabetes [8]. The benefit for reduction of mortality rate and HF events with dapagliflozin was significant in both subgroups suggesting that the effects of dapagliflozin on these endpoints is independent of $\mathrm{HbA}_{1 \mathrm{c}}$ [8]. We now recommend that in appropriate high-risk individuals with established type 2 diabetes, the decision to treat with a GLP-1 receptor agonist or SGLT2 inhibitor to reduce MACE, hHF, cardiovascular death or CKD progression should be considered independently of baseline $\mathbf{H b A}_{1 \mathbf{c}}$ or individualised $\mathbf{H b A}_{\mathbf{1 c}}$ target. That said, there are no specific analyses addressing $\mathrm{HbA}_{1 \mathrm{c}}<48 \mathrm{mmol} / \mathrm{mol}$ $(<6.5 \%)$. We continue to recommend that substituting a drug with known CVD, CKD and hHF benefit for one without known benefit in high-risk patients is reasonable when patients are at individualised glycaemic targets.

The Canagliflozin and Renal Events in Diabetes with Established Nephropathy Clinical Evaluation (CREDENCE) trial of the SGLT2 inhibitor canagliflozin was the first renal outcome trial of a diabetes medication [10] with a primary composite endpoint of end-stage kidney disease (dialysis, transplantation or a sustained eGFR of $<15 \mathrm{ml} \mathrm{min}^{-1}$ $[1.73 \mathrm{~m}]^{-2}$ ), a doubling of the serum creatinine level or death from renal or cardiovascular causes. The trial recruited patients with type 2 diabetes and CKD on the maximally tolerated dose of ACE inhibitors or angiotensin receptor blockers (ARBs) with a urinary albumin-to-creatinine ratio (UACR) of $300-5000 \mathrm{mg} / \mathrm{g}$ and an eGFR of 30 to $<90 \mathrm{ml} \mathrm{min}^{-1}[1.73 \mathrm{~m}]^{-2}$. This trial demonstrated a clear benefit of canagliflozin $(100 \mathrm{mg})$ on multiple renal endpoints, including progression to end-stage kidney disease, and on cardiovascular mortality, MACE and hHF. Furthermore, the CREDENCE results demonstrated that the benefits conferred by canagliflozin in terms of reducing MACE, hHF, cardiovascular mortality and renal endpoints were similar regardless of baseline status for cardiovascular or CKD grade 2-3 [11]. We now recommend that SGLT2 inhibitors should be used to prevent hHF, MACE and CV death and the progression of CKD in patients with type 2 diabetes with CKD. The benefits are clear-cut for those with UACR $>300 \mathrm{mg} / \mathrm{g}$ and eGFR $30-90 \mathrm{ml} \mathrm{min}^{-1}[1.73 \mathrm{~m}]^{-2}$ and less well established for lesser grades of CKD based on secondary endpoint analyses of the CVOT.

A concern in the CANVAS Program was the increased risk of amputation with canagliflozin compared with placebo [7]. In CREDENCE [10], although the risk of amputation was higher overall than in other SGLT2 inhibitor trials, no significant increase in risk was observed with canagliflozin $100 \mathrm{mg}$ vs placebo (HR 1.11, 95\% CI 0.79, 1.56). This may be due to the risk mitigation strategies employed: exclusion of patients with a history of a traumatic amputation within 12 months of screening, or an active foot ulcer, osteomyelitis, gangrene or critical ischaemia of the lower extremity within the 6 months of screening; and interruption of therapy for emergence of any of the above with careful consideration of the individual risks and benefits prior to restarting canagliflozin after resolution of the event. We now recommend that patients with foot ulcers or at high risk for amputation should only be treated with SGLT2 inhibitors after careful shared decision making around risks and benefits with comprehensive education on foot care and amputation prevention.

Based on the studies published thus far, we believe that for patients with type 2 diabetes and established atherosclerotic CVD (such as those with prior myocardial infarction, ischaemic stroke, unstable angina with ECG changes, myocardial ischaemia on imaging or stress test, or revascularisation of coronary, carotid, or peripheral arteries) where MACE is the gravest threat, that the level of evidence for MACE benefit is greatest for GLP-1 receptor agonists.

The Peptide Innovation for Early Diabetes Treatment 6 (PIONEER 6) cardiovascular safety trial of oral semaglutide, a GLP-1 receptor agonist, involved 3183 patients with type 2 diabetes followed for only a median of 16 months, but provided adequate demonstration of cardiovascular safety (HR 0.79 , $95 \%$ CI $0.57,1.11)$ and a strong signal for reduction of CV mortality rate (HR $0.49,95 \%$ CI $0.27,0.92)$ [12]. This formulation of semaglutide has been approved for marketing in the USA and a decision in the EU is expected soon.

For patients with or without established atherosclerotic CVD, but with HFrEF or CKD (eGFR 30 to $\leq 60 \mathrm{ml} \mathrm{min}^{-1}$ $[1.73 \mathrm{~m}]^{-2}$ or UACR $>30 \mathrm{mg} / \mathrm{g}$, particularly UACR $>300 \mathrm{mg} / \mathrm{g}$ ), the level of evidence for benefit is greatest for SGLT2 inhibitors. For patients with type 2 diabetes at low cardiovascular risk and without CKD, there have been no studies to examine the cardiovascular or renal benefit of GLP1 receptor agonists or SGLT2 inhibitors.

Some meta-analyses $[5,13,14]$ suggest the presence of heterogeneity in estimates for MACE and CV death with GLP-1 receptor agonists, although this is mostly due to the results of a single trial with lixisenatide. Likewise, there is some heterogeneity in the estimate for $\mathrm{CV}$ death with SGLT2 inhibitors. Whether differences in point estimates of benefits and harms are the result of differences in the effects of the medications, the design and conduct of the trials, or chance effects is uncertain. Attention to patient-specific factors and preferences, product labelling, meta-analyses, and the primary research reports should drive individualised clinical decision making with regards to prescribing particular medications within a class. For many patients, treatment with a GLP-1 receptor agonist or SGLT2 inhibitor in some healthcare settings involves considerable direct cost to them, and the impact of this on their overall wellbeing needs to be factored into decision making.

The Cardiovascular Outcome Study of Linagliptin vs Glimepiride in Type 2 Diabetes (CAROLINA) trial randomised adults at high cardiovascular risk to receive the 


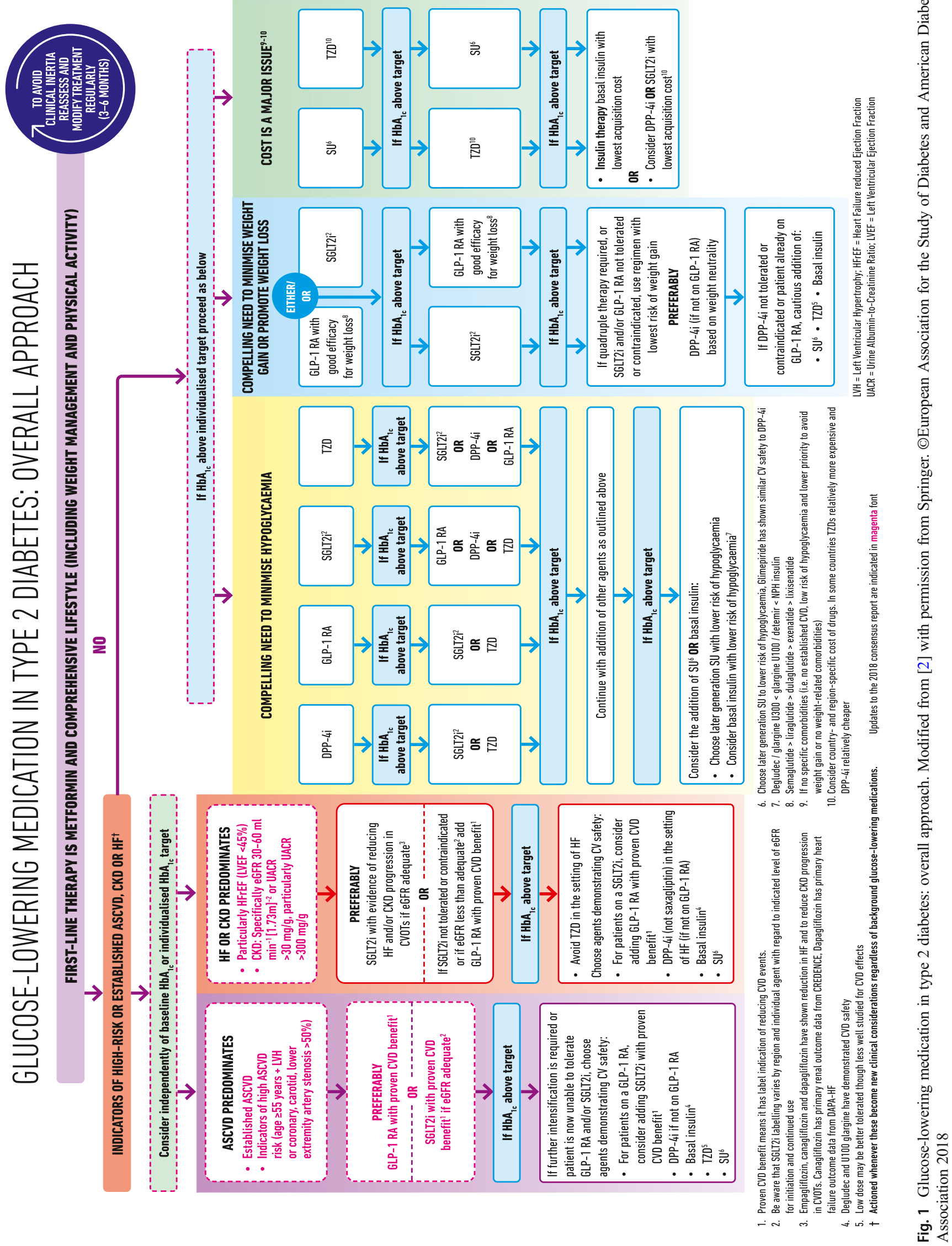




\section{CHOOSING GLUCOSE-LOWERING MEDICATION IN THOSE WITH INDICATORS OF HIGH-RISK OR ESTABLISHED ATHEROSCLEROTIC CARDIOVASCULAR DISEASE (ASCVD), CHRONIC KIDNEY DISEASE (CKD) OR HEART FAILURE (HF)}

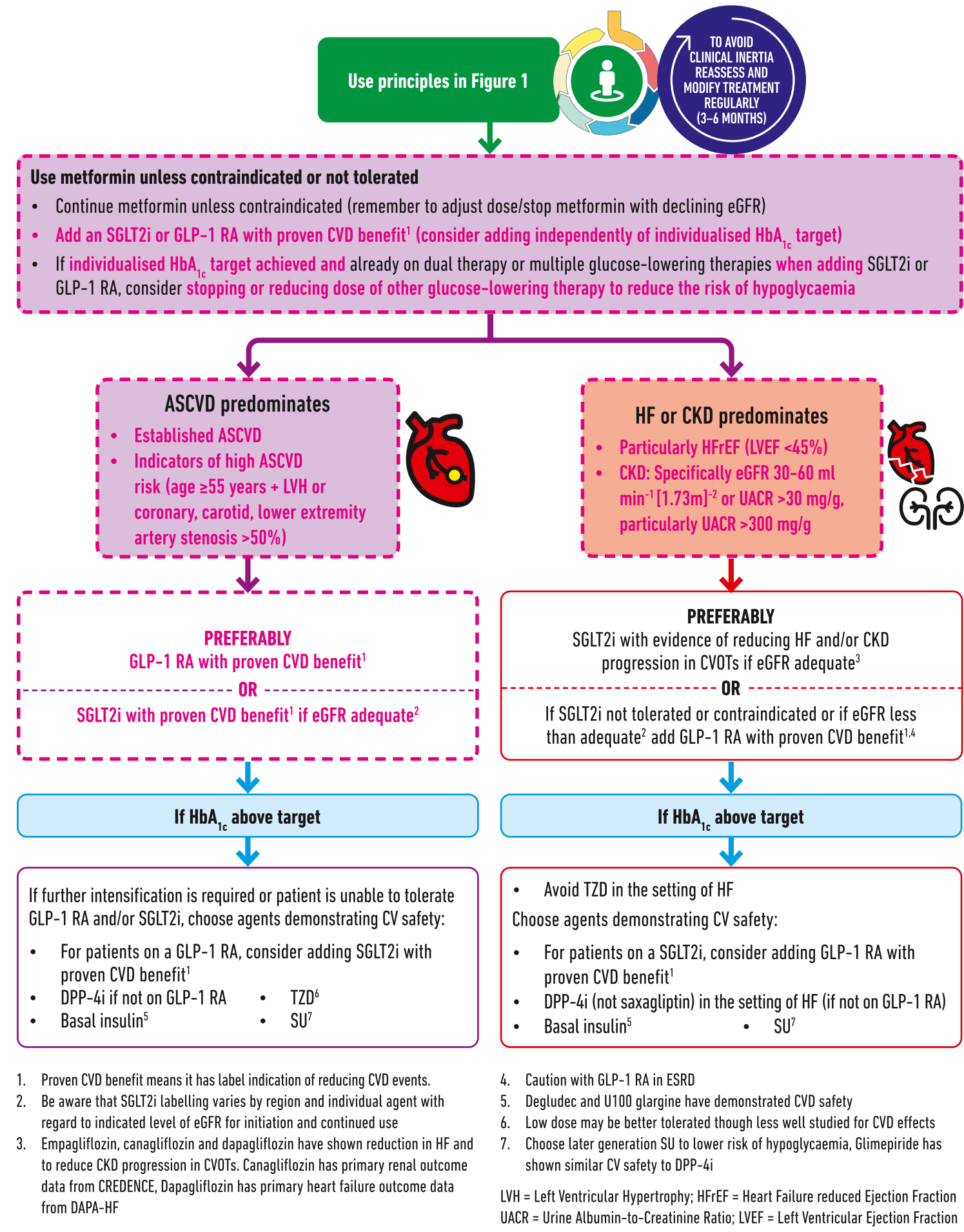

Updates to the 2018 consensus report are indicated in magenta font

Fig. 2 Glucose-lowering medication in type 2 diabetes: overall approach. Modified from [2] with permission from Springer. CEuropean Association for the Study of Diabetes and American Diabetes Association 2018 
dipeptidyl peptidase 4 (DPP-4) inhibitor linagliptin or to receive the sulfonylurea glimepiride to evaluate a primary MACE endpoint. No between-group difference in the primary endpoint was demonstrated (HR $0.98,95 \%$ CI $0.84,1.14$ ). At trial end, for linagliptin as compared with glimepiride, there was a $1.5 \mathrm{~kg}$ weight loss benefit, no difference in $\mathrm{HbA}_{1 \mathrm{c}}$ or introduction of glucose-lowering medications post-baseline, and substantial benefits in terms of reductions in hypoglycaemia, though serious hypoglycaemic events were rare with glimepiride $(0.45 / 100$ patient-years) [15]. Paired with other DPP-4 inhibitor CVOTs, including Cardiovascular and Renal Microvascular Outcome Study with Linagliptin (CARMELINA) [16], which demonstrated the CV safety of linagliptin, this is a reassuring safety signal for glimepiride, an inexpensive and effective sulfonylurea. It is unclear whether these findings extend to other sulfonylureas.

Whereas we previously stated that there was limited evidence for initial combination therapy, the Vildagliptin Efficacy in Combination with Metformin for Early Treatment of Type 2 Diabetes (VERIFY) trial provides additional information. The initial combination of the DPP-4 inhibitor vildagliptin and metformin was shown to provide for a lower rate of secondary failure of glycaemic control to $\mathrm{HbA}_{1 \mathrm{c}} \geq 53 \mathrm{mmol} / \mathrm{mol}(\geq 7 \%)$ vs metformin alone or the sequential addition of metformin and vildagliptin [17]. We now suggest that providers should engage in shared decision making around initial combination therapy in newonset cases of type 2 diabetes.

There are several major questions regarding the optimal application of new diabetes drugs. One obvious question arising from recent trial results is whether combined use of GLP-1 receptor agonists and SGLT2 inhibitors provides additional benefit for the prevention of MACE, CV death, hHF and CKD progression. Three trials have demonstrated the $\mathrm{HbA}_{1 \mathrm{c}}$-lowering and weight-reduction efficacy of the combination [18-20], but none addresses the impact of the combination of the two on cardiorenal endpoints. A second question that arises from the recent secondary analyses of SGLT2 inhibitor studies is whether there are subsets of patients who benefit disproportionately, or very little, from treatment with the newer diabetes drugs. The emerging evidence that SGLT2 inhibitors may be particularly useful in preventing adverse outcomes in patients with diabetes with HFrEF raises the possibility of more targeted use of these agents. Finally, the mechanism(s) of action by which GLP-1 receptor agonists and SGLT2 inhibitors confer cardiorenal benefit in diabetes are not understood. Research in this area will be very useful in optimising the now clear potential of drugs for diabetes to mitigate the cardiovascular and renal complications of the disease. Modifications to the main figures of the prior publication are suggested as shown in Figs 1 and 2.
Acknowledgements The authors would like to thank William T. Cefalu (Director of the Division of Diabetes, Endocrinology, and Metabolic Diseases at the National Institute of Diabetes and Digestive and Kidney Diseases, National Institutes of Health) for his review. The authors would like to acknowledge M. Saraco (Managing Director, Scientific \& Medical Affairs) from ADA, as well as M. Hata (Executive Assistant) and P. Niemann (Executive Assistant) from EASD for their assistance.

The authors would also like to acknowledge M. Bonar (Creative Director) and C. Franklin (Design Assistant) from the Leicester Diabetes Centre, Leicester, UK, who provided considerable support in drafting and amending the figures.

The authors also acknowledge the careful review and helpful suggestions of the members of the ADA Professional Practice Committee and the EASD Committee on Clinical Affairs.

Funding This activity was funded by the American Diabetes Association and the European Association for the Study of Diabetes.

Duality of interest M.J. Davies reports personal fees and grants from Boehringer Ingelheim, Janssen, Novo Nordisk and Sanofi, and personal fees from AstraZeneca, Eli Lilly, Gilead Sciences Ltd., Intarcia/Servier, Merck Sharp \& Dohme, Mitsubishi Tanabe Pharma Corporation and Takeda Pharmaceuticals International Inc.

D. A. D'Alessio reports personal fees from Eli Lilly, Merck, Novo Nordisk and Intarcia, and grants from Merck and Ligand during the conduct of the study; personal fees from Lilly Merck, Novo Nordisk and Intarcia, and grants from Merck and Ligand outside the submitted work.

C. Mathieu reports grants and fees from Novo Nordisk, grants and fees from Sanofi, grants and fees from Merck Sharp \& Dohme, grants and fees from Eli Lilly and Company, grants and fees from Novartis, fees from AstraZeneca, grants and fees from Boehringer Ingelheim, fees from Roche Diagnostics, grants and fees from Medtronic, and grants and fees from ActoBio Therapeutics outside the submitted work, with all fees paid to her university.

G. Mingrone reports grants and personal fees from Novo Nordisk, personal fees from Johnson \& Johnson, personal fees from Fractyl Inc., during the conduct of the study.

P. Rossing reports grants, non-financial support and other from Novo Nordisk, grants and other from Astra Zeneca, other from Bayer, other from Boehringer Ingelheim, other from MSD, other from Eli Lilly, during the conduct of the study.

A. Tsapas reports non-financial support from the European Association for the Study of Diabetes, during the conduct of the study; grants and other from Boehringer Ingelheim, grants and other from Novo Nordisk, other from Novartis, grants and other from Sanofi, grants and other from AstraZeneca, grants from GSK, grants and other from European Foundation for the Study of Diabetes (EFSD), outside the submitted work.

D. J. Wexler reports serving on a Data Monitoring Committee for Novo Nordisk

J. B. Buse has provided consultation to Adocia, AstraZeneca, Eli Lilly, MannKind, NovaTarg, Novo Nordisk, Senseonics and vTv Therapeutics with fees paid to the University of North Carolina. He has received grant support from Novo Nordisk, Sanofi and vTv Therapeutics. He is a consultant to Cirius Therapeutics Inc., CSL Behring, Neurimmune AG. He holds stock options in Mellitus Health, PhaseBio, Stability Health and Pendulum Therapeutics. He is supported by a grant from the National Institutes of Health (UL1TR002489).

Contribution statement All authors were responsible for drafting the article and revising it critically for important intellectual content. All authors approved the version as published. 


\section{References}

1. Davies MJ, D'Alessio DA, Fradkin J et al (2018) Management of hyperglycemia in type 2 diabetes, 2018. A consensus report by the American Diabetes Association (ADA) and the European Association for the Study of Diabetes (EASD). Diabetes Care 41(12):2669-2701. https://doi.org/10.2337/dci18-0033

2. Davies MJ, D'Alessio DA, Fradkin J et al (2018) Management of hyperglycaemia in type 2 diabetes, 2018. A consensus report by the American Diabetes Association (ADA) and the European Association for the Study of Diabetes (EASD). Diabetologia. 61(12):2461-2498. https://doi.org/10.1007/s00125-018-4729-5

3. Gerstein HC, Colhoun HM, Dagenais GR et al (2019) Dulaglutide and cardiovascular outcomes in type 2 diabetes (REWIND): a double-blind, randomised placebo-controlled trial. Lancet. 394(10193):121-130. https://doi.org/10.1016/S0140-6736(19) 31149-3

4. Wiviott SD, Raz I, Bonaca MP et al (2019) Dapagliflozin and cardiovascular outcomes in type 2 diabetes. N Engl J Med 380(4):347-357. https://doi.org/10.1056/NEJMoa1812389

5. Zelniker TA, Wiviott SD, Raz I et al (2019) Comparison of the effects of glucagon-like peptide receptor agonists and sodiumglucose cotransporter 2 inhibitors for prevention of major adverse cardiovascular and renal outcomes in type 2 diabetes mellitus. Circulation. 139(17):2022-2031. https://doi.org/10.1161/ CIRCULATIONAHA.118.038868

6. Kato ET, Silverman MG, Mosenzon O et al (2019) Effect of dapagliflozin on heart failure and mortality in type 2 diabetes mellitus. Circulation. 139(22):2528-2536. https://doi.org/10.1161/ CIRCULATIONAHA.119.040130

7. Figtree GA, Rådholm K, Barrett TD et al (2019) Effects of canagliflozin on heart failure outcomes associated with preserved and reduced ejection fraction in type 2 diabetes mellitus. Circulation. 139(22):2591-2593. https://doi.org/10.1161/ CIRCULATIONAHA.119.040057

8. McMurray JJV, Solomon SD, Inzucchi SE et al (2019) Dapagliflozin in patients with heart failure and reduced ejection fraction. N Engl J Med. https://doi.org/10.1056/NEJMoa1911303

9. Inzucchi SE, Kosiborod M, Fitchett D et al (2018) Improvement in cardiovascular outcomes with empagliflozin is independent of glycemic control. Circulation. 138(17):1904-1907. https://doi.org/ 10.1161/CIRCULATIONAHA.118.035759

10. Perkovic V, Jardine MJ, Neal B et al (2019) Canagliflozin and renal outcomes in type 2 diabetes and nephropathy. N Engl J Med 380(24):2295-2306. https://doi.org/10.1056/NEJMoa1811744

11. Mahaffey KW, Jardine MJ, Bompoint S et al (2019) Canagliflozin and cardiovascular and renal outcomes in type 2 diabetes mellitus and chronic kidney disease in primary and secondary cardiovascular prevention groups. Circulation. 140(9):739-750. https://doi.org/ 10.1161/CIRCULATIONAHA.119.042007

12. Husain M, Birkenfeld AL, Donsmark M et al (2019) Oral semaglutide and cardiovascular outcomes in patients with type 2 diabetes. N Engl J Med 381(9):841-851. https://doi.org/10.1056/ NEJMoa1901118

13. Giugliano D, Maiorino MI, Bellastella G, Longo M, Chiodini P, Esposito K (2019) GLP-1 receptor agonists for prevention of cardiorenal outcomes in type 2 diabetes: an updated meta-analysis including the REWIND and PIONEER 6 trials. Diabetes Obes Metab 21(11):2576-2580. https://doi.org/10.1111/dom.13847

14. Kristensen SL, Rørth R, Jhund PS et al (2019) Cardiovascular, mortality, and kidney outcomes with GLP-1 receptor agonists in patients with type 2 diabetes: a systematic review and metaanalysis of cardiovascular outcome trials. Lancet Diabetes Endocrinol 7(10):776-785. https://doi.org/10.1016/S22138587(19)30249-9

15. Rosenstock J, Kahn SE, Johansen OE et al (2019) Effect of linagliptin vs glimepiride on major adverse cardiovascular outcomes in patients with type 2 diabetes: the CAROLINA randomized clinical trial. JAMA 322(12):1155. https://doi.org/10. 1001/jama.2019.13772

16. Rosenstock J, Perkovic V, Johansen OE et al (2019) Effect of linagliptin vs placebo on major cardiovascular events in adults with type 2 diabetes and high cardiovascular and renal risk: the CARMELINA randomized clinical trial. JAMA 321(1):69-79. https://doi.org/10.1001/jama.2018.18269

17. Matthews DR, Paldánius PM, Proot P, Chiang YT, Stumvoll M, del Prato S (2019) Glycaemic durability of an early combination therapy with vildagliptin and metformin versus sequential metformin monotherapy in newly diagnosed type 2 diabetes (VERIFY): a 5year, multicentre, randomised, double-blind trial. Lancet 394(10208):1519-1529. https://doi.org/10.1016/S0140-6736(19) 32131-2

18. Zinman B, Bhosekar V, Busch R et al (2019) Semaglutide once weekly as add-on to SGLT-2 inhibitor therapy in type 2 diabetes (SUSTAIN 9): a randomised, placebo-controlled trial. Lancet Diabetes Endocrinol 7(5):356-367. https://doi.org/10.1016/ S2213-8587(19)30066-X

19. Ludvik B, Frias JP, Tinahones FJ et al (2018) Dulaglutide as add-on therapy to SGLT-2 inhibitors in patients with inadequately controlled type 2 diabetes (AWARD-10) : a 24- week, randomized, double-blind, placebo-controlled trial. Lancet Diabetes Endocrinol 6(5):370-381. https://doi.org/10.1016/S2213-8587(18)30023-8

20. Frías JP, Guja C, Hardy E et al (2016) Exenatide once weekly plus dapagliflozin once daily versus exenatide or dapagliflozin alone in patients with type 2 diabetes inadequately controlled with metformin monotherapy (DURATION-8): a 28 week, multicentre, double-blind, phase 3, randomised controlled trial. Lancet Diabetes Endocrinol 4(12):1004-1016. https://doi.org/10.1016/ S2213-8587(16)30267-4

Publisher's note Springer Nature remains neutral with regard to jurisdictional claims in published maps and institutional affiliations. 